\title{
Industrielle Energietechnik
}

\author{
Gregor Berger, Alexandra Holzer und Harald Raupenstrauch
}

Lehrstuhl für Thermoprozesstechnik, Montanuniversität Leoben, Leoben, Österreich

Eingegangen 30. April 2020; angenommen 22. Mai 2020; online publiziert 8. Juni 2020

Zusammenfassung: Energieeffizienz, Prozessoptimierung und die damit einhergehende Ressourcenschonung sind in Hinblick auf ökonomische, ökologische und sozioökonomische Aspekte ein zentrales Thema der energieintensiven Industrie. Die Arbeitsgruppe „Industrielle Energietechnik" am Lehrstuhl für Thermoprozesstechnik (TPT) beschäftigt sich in diesem Zusammenhang mit der Bewertung von Prozessen und Erarbeitung von technisch-wirtschaftlichen Maßnahmen mit dem Ziel einer Energieeffizienzsteigerung. Im Speziellen handelt es sich um die energetische Optimierung von thermischen Prozessen und Anlagen im Hochtemperaturbereich (z. B. Industrieöfen, Hochtemperaturreaktoren, etc.) und deren Einbindung in den Gesamtprozess.

Neben der Bilanzierung von Energie- und Stoffströmen sowie der Bewertung der Energieeffizienz verfügt die Arbeitsgruppe über Kompetenzen im Bereich der Erfassung wichtiger Betriebsparameter in Prozessen. So wurden mittels geeigneter Ausstattungen in der Vergangenheit zahlreiche Temperaturerfassungen bis hin zu In-situ-Messungen durchgeführt.

Schlüsselwörter: Industrielle Energietechnik, Energieeffizienzsteigerung, Ressourcenschonung, Energieintensive Industrie

\section{Industrial Energy Technology}

Abstract: Energy efficiency, process optimization, and the associated conservation of resources are-regarding the economic, ecological, and socio-economic aspects-a central topic of the energy-intensive industry. In this context, the working group "Industrial Energy Technology" at the Chair of Thermal Processing Technology (TPT) deals with the evaluation of processes and the development of technical-economic measures with the aim of increasing energy

$\overline{\text { Dipl.-Ing. G. Berger }(\bowtie)}$

Lehrstuhl für Thermoprozesstechnik,

Montanuniversität Leoben,

Franz-Josef-Straße 18,

8700 Leoben, Österreich

gregor.berger@unileoben.ac.at efficiency. In particular, this involves the energetic optimization of thermal processes and plants in high temperature applications (e.g. industrial furnaces, high temperature reactors, etc.) and their integration into the overall process.

In addition to balancing the energy and material flows and evaluation of energy efficiency, the working group has competences in the field of measuring important operating parameters in processes. In the past, numerous temperature monitoring up to in-situ measurements have been carried out using suitable equipment.

Keywords: Industrial energy engineering, Energy efficiency, Resource conservation, Energy-intensive industry

\section{Einleitung}

Die Arbeitsgruppe für industrielle Energietechnik beschäftigt sich mit der Steigerung der Energieeffizienz von Industrieöfen bzw. gesamten Prozessrouten in der energieintensiven Industrie. Dabei werden Industriebetriebe bei der Detektion und Analyse anfallender thermischer Verluste unterstützt und darauf basierend Möglichkeiten zur Vermeidung bzw. Nutzung derer in anderen Prozessen entwickelt.

In Zusammenarbeit mit weiteren Forschungseinheiten am Lehrstuhl für Thermoprozesstechnik (TPT) werden auch andere Fragestellung der Energietechnik bearbeitet. Als Beispiel dient in diesem Zusammenhang die kooperative Untersuchung der Auswirkungen eines erhöhten Wasserstoffanteils im Erdgas auf die Industrie mit der Abteilung für Modellierung und Simulation.

\section{Kompetenzen und Ausstattung}

Durch Bilanzierung von Stoff- und Energieströmen kann eine fundierte Aussage über die Energieeffizienz eines Prozesses getroffen werden. Diese liefert die Basis für weitere Detailanalysen, die in weiterer Folge zur Ableitung effizienter Maßnahmen führen. Zusammen mit dem jeweiligen In- 
dustriepartner kann somit beurteilt werden, welche weiterführenden Schritte am Weg zur energie- und ressourceneffizienten Produktion notwendig sind.

Essenziell für jegliche energetische Betrachtung ist eine korrekte Datenerfassung, welche als Grundlage zur Erkennung und Berechnung möglicher Einsparungspotentiale dient. Da fehlerhafte Messparameter die Ergebnisse negativ beeinflussen und in Folge dessen zu Falschaussagen führen, stellt die messtechnische Erfassung von Betriebsparametern einen der wichtigsten Arbeitsschritte dar. Um eine exakte Bestimmung der notwendigen Parameter zu gewährleisten, verfügt der Lehrstuhl für Thermoprozesstechnik über geeignetes Messequipment, wovon eine Auswahl in den folgenden Kapiteln näher beschrieben wird.

\subsection{Absaugpyrometer}

Das Erfassen der Temperatur von Ofenatmosphären ist mit einigen Schwierigkeiten verbunden, da heiße Oberflächen durch ihre abgestrahlte Wärme zu einer Verfälschung des Messergebnisses führen. Wird ein herkömmliches Thermoelement in einem Ofen zur Messung der Gastemperatur angebracht, beeinflusst die Wärmestrahlung der heißen Ofenwände die erfasste Temperatur. Ein sogenanntes Absaugpyrometer ermöglicht eine direkte Erfassung der Temperatur eines Gasstroms ohne den Einfluss der Strahlungswärme. Dabei wird die Ofenatmosphäre abgesaugt und über ein Thermoelement, das durch mehrere Keramik-Schutzrohre vom Strahlungseinfluss abgeschirmt ist, geleitet. Ist der Absaugvolumenstrom ausreichend groß, wird die von den Keramikrohren übertragene Wärme konvektiv abgeführt und die Gleichgewichtstemperatur des Thermoelements entspricht der Temperatur der abgesaugten Gase.

In Abb. 1 ist ein Absaugpyrometer dargestellt, welches zur Messung der Gastemperatur im Hochtemperaturbereich bis ca. $1600^{\circ} \mathrm{C}$ verwendet werden kann.

\subsection{Abgasanalysegerät}

Das bei Verbrennungsprozessen ausgestoßene Abgas enthält Wärme, die dem Prozess nicht zur Verfügung steht. Die gleichzeitig emittierten Schadstoffe (z. B. $\mathrm{CO}_{2}, \mathrm{CO}, \mathrm{NO}_{\mathrm{x}}$, Ruß) belasten die Umwelt und haben einen negativen Einfluss auf das Klima. Mit dem in Abb. 2 dargestelltem Abgasanalysegerät können emittierte Schadstoffe und die im

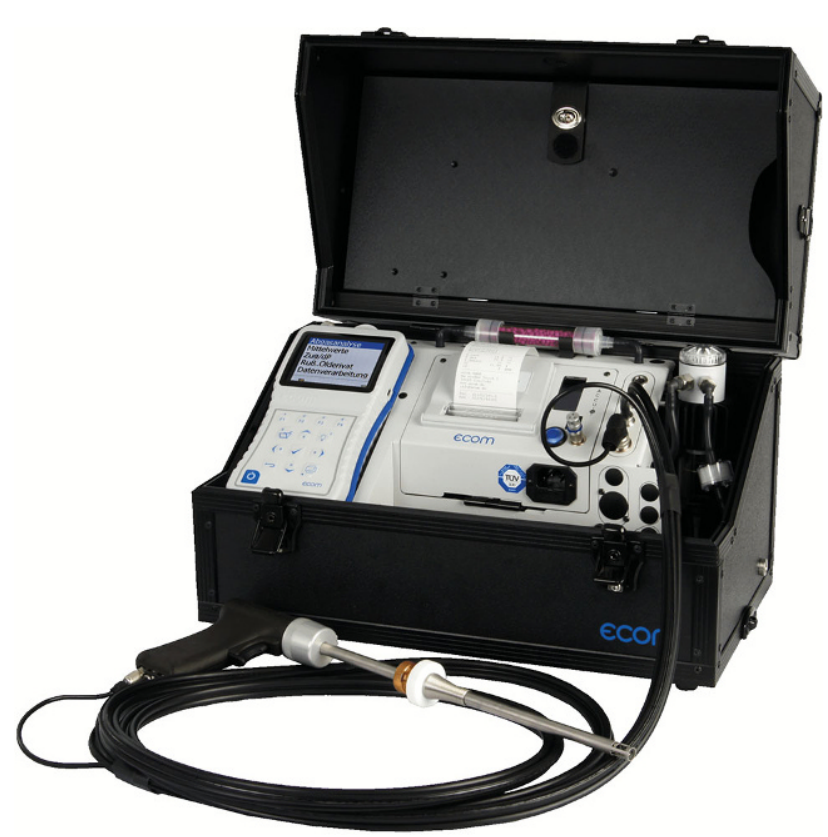

Abb. 2: Abgasanalysegerät ecom J2KNpro [2]

Abgas enthaltene Energie erfasst werden. Es können ebenfalls Aussagen über die Vollständigkeit der Verbrennung, den Luftüberschuss und den thermischen Abgasverlust getätigt werden.

\subsection{Thermografiesystem}

Für eine berührungslose Temperaturerfassung steht ein Thermografiesystem zur Verfügung (Abb. 3). Dieses findet in den verschiedensten industriellen Anwendungsgebieten der Entwicklung, Fertigung und Prozessüberwachung Einsatz. Voraussetzung für eine korrekte Temperaturerfassung ist die Verwendung des korrekten Emissionskoeffizienten. Dieser ist material- und temperaturabhängig und muss vor der Messung bestimmt werden. Dabei wird die Oberflächentemperatur mit einem Thermoelement und die Strahlungstemperatur eines Körpers mit dem Thermografiesystem erfasst. Mit den beiden erfassten Temperaturen kann der temperaturabhängige Emissionskoeffizient berechnet werden.
Abb. 1: Schematische Darstellung eines Absaugpyrometers zur Erfassung von Gastemperaturen [1]

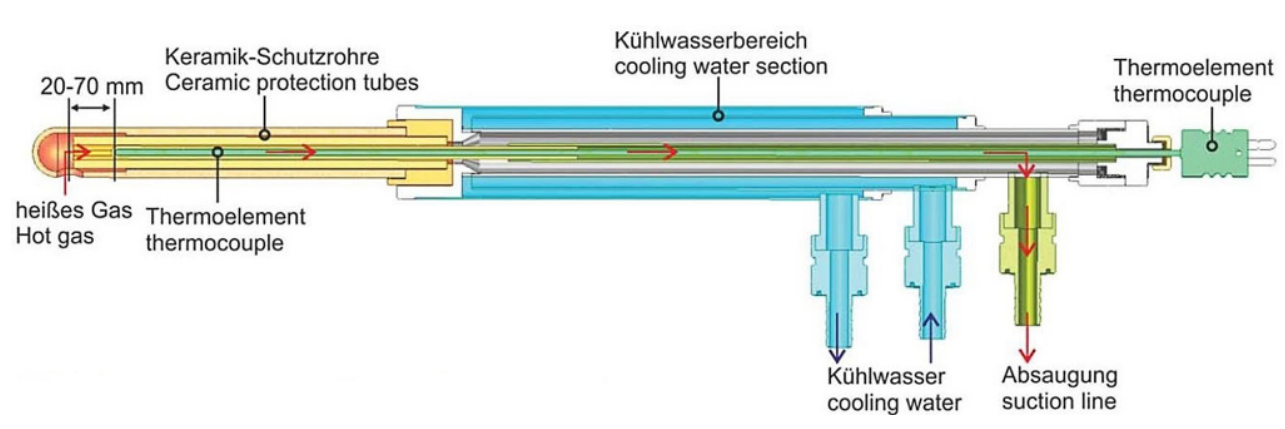


Abb. 3: Thermografiesystem VarioCAM hrhead [3] (a) und Einsatz der bildgebenden Temperaturerfassung (b)
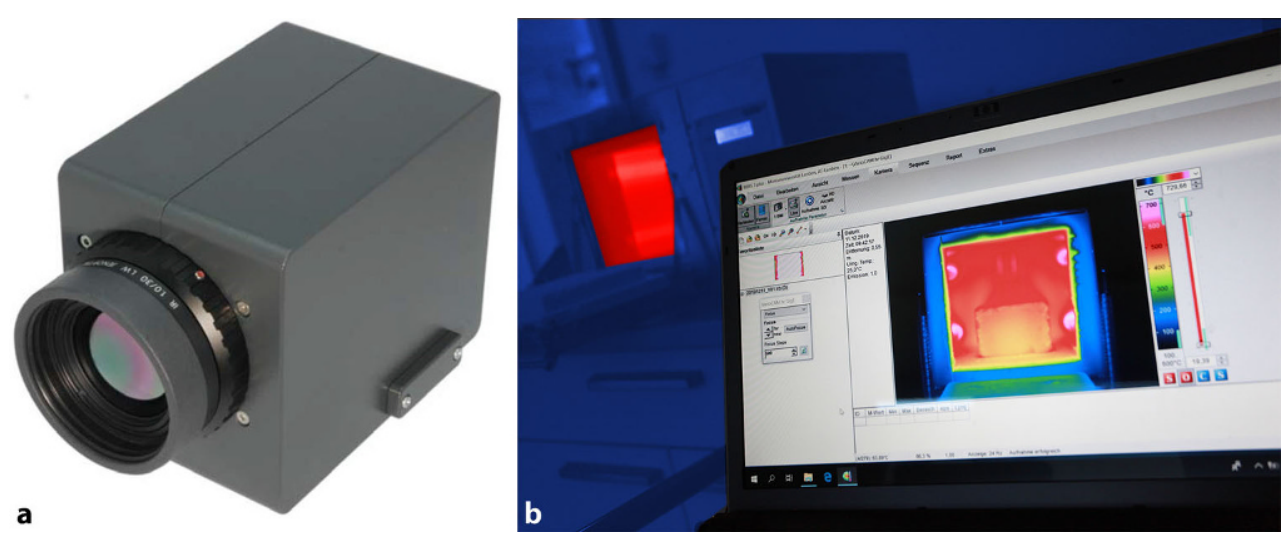

\section{Projekte}

Zur Verdeutlichung der vorhandenen Kompetenzen werden im folgenden Abschnitt aktuell laufende Projekte der Arbeitsgruppe beschrieben. Parallel zu diesen Betätigungsfeldern werden Abschlussarbeiten in den Bereichen Abwärmenutzung, Klärschlammmonoverwertung und Methanemissionen in Erdgasnetzen betreut.

\subsection{Entwicklung eines Prozess- und Bewertungs- modells zur Beurteilung der Energieeffizienz industrieller Prozesse}

Ziel dieses Projektes ist die Entwicklung eines Prozess- und Bewertungsmodells zur nachhaltigen Erhöhung der Energieeffizienz industrieller Prozesse. Erste Erfahrungen mit einem solchen, auf MS Excel basierten, Modell wurden im Projekt EneffGieß im Bereich der Gießereiindustrie gesammelt [4].

In einem darauf aufbauenden Forschungsvorhaben soll im Konsortium TÜV Süd Landesgesellschaft Österreich (TÜV Süd) und TPT neben der Gießereiindustrie eine Funktionserweiterung auf die Zement- und Glasindustrie erfolgen.

Das entwickelte Modell soll zum einen der Abbildung und Bewertung des betrieblichen Status-Quo dienen, zum anderen soll es die Möglichkeit einer Simulation der Implementierung neuer Technologien in den Prozess bieten. Daraus resultierend werden die Auswirkungen ebensolcher auf die Gesamtenergieeffizienz des Prozesses aufgezeigt und die Ableitung von Empfehlungen für prozessbedingte Verbesserungen wird ermöglicht. Speziell in energieintensiven Branchen führen technisch-wirtschaftliche Maßnahmen in der Regel zu deutlichen Energieeinsparungen.

Mit der Entwicklung ebendieser branchenspezifischen Bewertungsmodelle wird es dem TÜV Süd Landesgesellschaft Österreich zukünftig möglich sein, für jeden Zement-, Glas- und Gießereibetrieb eine Bewertung des betrieblichen Energieverbrauchs mittels bottom-up Analyse durchzuführen. Dabei können gesamte Prozessrouten bzw. daraus resultierende Produkte betrachtet und bewertet werden. Die Erweiterung des Produktportfolios um diese Dienstleistung ist seitens TÜV Süd bereits in Planung.
3.2 Greening the Gas - Verbrennungstechnische und sicherheitsrelevante Anforderungen in Hinblick auf einen erhöhten Biogas- und Wasserstoffgehalt im Erdgas

Für die Umsetzung der österreichischen Klimaziele sind zahlreiche Forschungsaktivitäten notwendig. Um die Energieversorgung über das Erdgasnetz ökologischer zu gestalten, wurde von der Österreichischen Vereinigung für das Gas- und Wasserfach (ÖVGW) das Projekt "Greening the Gas" ins Leben gerufen. Ziel ist es, einen technischen Ordnungsrahmen zu schaffen, der die Integration von Biogas und Wasserstoff in die bestehenden Gasnetze zulässt. Im Zuge dieses Großprojekts wurde der Lehrstuhl für Thermoprozesstechnik von der ÖVGW damit beauftragt, die verbrennungstechnischen und sicherheitsrelevanten Anforderungen in Hinblick auf einen erhöhten Biogas- und Wasserstoffgehalt im Erdgasnetz zu untersuchen.

Eine Zumischung von den genannten alternativen Gasen hat vor allem auf Industrieprozesse große Auswirkungen. Speziell in metallurgischen Wärmebehandlungsprozessen werden hohe Anforderungen an eine konstante Ofenatmosphäre gestellt. Eine Änderung der Erdgasqualität beeinflusst ebendiese und führt in weiterer Folge zur Veränderung der Produktqualität. Darauf begründet sich die Limitierung des Wasserstoffgehalts im Erdgasnetz auf aktuell 4 vol.-\%. Die Untersuchungen des TPT zielen darauf ab, Industrieprozesse auf ihre Empfindlichkeit bezüglich Veränderungen der Erdgasqualität zu analysieren und neue Grenzwerte für die Einspeisung von alternativen Gasen zu bestimmen. Dabei wurde neben den verbrennungstechnischen Eigenschaften neuer Gasmischungen auch auf sicherheitstechnische Aspekte eingegangen. Schlussendlich wird anhand einer technoökonomischen Analyse die zeitliche Umsetzbarkeit von höheren Wasserstoffanteilen im Erdgasnetz bestimmt [5].

\subsection{Machbarkeitsstudie FlashMelt}

Der Glasschmelzprozess ist aufgrund der hohen Temperaturen sehr energieintensiv. Um eine geläuterte und homogene Glasschmelze zu erhalten, sind Temperaturen von über $1400^{\circ} \mathrm{C}$ erforderlich. Die heute in den meisten Glas- 
hütten verwendeten Schmelzwannen basieren auf einem 140 Jahre alten Prinzip. Aufgrund der Konstruktion treten bei diesen Schmelzwannen rückwärts laufende Strömungswalzen auf, die die Aufenthaltszeit der Glasschmelze in der Wanne deutlich verlängern. Während die minimale Zeit für das Schmelzen von Glas im Labormaßstab 3 bis $5 \mathrm{~h}$ beträgt, weisen Schmelzwannen eine durchschnittliche Aufenthaltszeit von 20 bis $60 \mathrm{~h}$ auf. Das führt aufgrund der hohen Temperaturen zu hohen Wärmeverlusten über die Oberfläche der Schmelzwanne [6].

Um den Prozess des Glasschmelzens energieeffizienter zu gestalten, plant der Lehrstuhl für Thermoprozesstechnik, ein innovatives Aggregat für das Schmelzen von Glas zu entwerfen. Unter der Voraussetzung eines ausreichenden Wärmeeintrags, einem minimalen Staubaustrag und der Möglichkeit der zusätzlichen elektrischen Beheizung sieht das Konzept einen modifizierten Flugstromreaktor vor, der bei gleichbleibender Glasqualität kosten- und energieeffizienter Glasschmelzen produzieren kann. Im Zuge einer Machbarkeitsstudie werden sämtliche Aspekte dieser Überlegung betrachtet.

\section{Schlussfolgerung und Zusammenfassung}

Die aktuell laufenden Projekte in der Arbeitsgruppe für industrielle Energietechnik haben für die energieintensive Industrie auf dem Weg in Richtung gesteigerter Ressourcenund Energieeffizienz hohe Zukunftsrelevanz. Die Energieeffizienzsteigerung der industriellen Produktionsprozesse sowie die damit einhergehende Verringerung der thermischen Verluste sind von wesentlicher Bedeutung bei der Erreichung der angepeilten Klima- und Energieziele. Wichtig in diesem Zusammenhang ist stets eine gezielte und anwendungsnahe Forschungs- und Entwicklungstätigkeit, welche in enger Kooperation mit dem jeweiligen Industriepartner erfolgt. Dadurch kann gewährleistet werden, dass den Anforderungen der Industrie entsprochen wird und die entwickelten Technologien kompatibel mit den zugrundeliegenden Produktionsprozessen sind. Weitere Vorteile dieser Synergie sind das praxisorientierte Vorantreiben der Forschung im Bereich industrielle Energietechnik und die Unterstützung der energieintensiven Industriebranchen auf ihrem Weg in die Energiezukunft des 21. Jahrhunderts. In diesem Zusammenhang ist der Lehrstuhl für Thermoprozesstechnik stets bemüht, ein verlässlicher Partner zu sein.

Funding. Open access funding provided by Montanuniversität Leoben.

Open Access Dieser Artikel wird unter der Creative Commons Namensnennung 4.0 International Lizenz veröffentlicht, welche die Nutzung, Vervielfältigung, Bearbeitung, Verbreitung und Wiedergabe in jeglichem Medium und Format erlaubt, sofern Sie den/die ursprünglichen $\mathrm{Au}$ tor(en) und die Quelle ordnungsgemäß nennen, einen Link zur Creative Commons Lizenz beifügen und angeben, ob Änderungen vorgenommen wurden.

Die in diesem Artikel enthaltenen Bilder und sonstiges Drittmaterial unterliegen ebenfalls der genannten Creative Commons Lizenz, sofern sich aus der Abbildungslegende nichts anderes ergibt. Sofern das betreffende Material nicht unter der genannten Creative Commons Lizenz steht und die betreffende Handlung nicht nach gesetzlichen Vorschriften erlaubt ist, ist für die oben aufgeführten Weiterverwendungen des Materials die Einwilligung des jeweiligen Rechteinhabers einzuholen.

Weitere Details zur Lizenz entnehmen Sie bitte der Lizenzinformation auf http://creativecommons.org/licenses/by/4.0/deed.de.

\section{Literatur}

1. Paul Gohte GmbH: Hinweise Absaugpyrometer, Bochum, 2018

2. ecom GmbH: Bedienungsanleitung: ecom J2KNpro, Iserlohn, 2019

3. InfraTec GmbH: Benutzerhandbuch für das Thermografiesystem VarioCAM hr head, Dresden, 2015

4. Egger, D.; Rauter, M.; Raonic, Z.; Raupenstrauch, H.: EnEffGieß: Entwicklung eines Life-Cycle orientierten Ansatzes zur Bewertung energieeffizienter, nachhaltiger Giessereiprodukte - Bottom-Up Betrachtung, Gießerei Rundschau, 64 (2017), S. 12-17

5. Raupenstrauch, $\mathrm{H}$.; et al.: Projekt Greening the Gas: Verbrennungstechnische und sicherheitsrelevante Anforderungen in Hinblick auf einen erhöhten Biogas- und Wasserstoffanteil im Erdgas, Montanuniversität, Leoben, 2019

6. Beerkens, R.: New Concepts for Energy Efficient \& Emission Friendly Melting of Glass, in: Varshneya, A. K.; Schaeffer, H. A.; Richardson, K. A.; Wightman, M.; Pye, L. D. (Hrsg.): Processing, Properties, and Applications of Glass and Optical Materials, Ceramic Transactions Series, Hoboken, NJ, USA: John Wiley \& Sons, Inc, 2012, S. $1-23$

Hinweis des Verlags. Der Verlag bleibt in Hinblick auf geografische Zuordnungen und Gebietsbezeichnungen in veröffentlichten Karten und Institutsadressen neutral. 